\title{
Barriers and facilitators to nutritional risk screening of older adults in primary care
}

\author{
Christine Mills \\ Queen's University, Kingston, Canada
}

\begin{abstract}
Introduction: Over one-third of these community-dwelling older adults in Canada are at increased nutritional risk. Worldwide, two-thirds of older adults are at increased nutritional risk, although this figure includes those who are hospitalized or in long term care. Nutritional risk can lead to malnutrition; this occurs when an individual's food intake has an imbalance of energy, protein, or other nutrients. Nutritional risk and malnutrition are associated with poor quality of life, increased hospitalization, and premature mortality. Since malnutrition starts in the community, primary care is the ideal location for nutritional risk screening. If nutritional risk is identified early, before it progresses to malnutrition, it can be more easily treated. It is therefore important to understand barriers and facilitators to nutritional risk screening in primary care.
\end{abstract}

Materials and Methods: The peer-reviewed and grey literature were searched. The databases CINAHL, Embase, Medline, and Google Scholar were used to identify articles related to barriers and facilitators to nutritional risk screening of older adults in primary care. A Google search identified publications from the grey literature related to nutritional risk screening of older adults. Key informants consisting of health care professionals working in primary care were asked to identify additional barriers. The Theoretical Domains Framework (TDF) was used to classify the barriers and facilitators.

Results: Nine barriers and nine facilitators relating to nutritional risk screening of older adults in primary care were identified. These barriers and facilitators were located within the following domains of the TDF: knowledge; skills; social/professional role and identity; beliefs about capabilities; beliefs about consequences; motivation and goals; memory, attention and decision processes; environmental context and resources; social influences; emotions; behavioural regulation; and nature of the behaviours.

Discussion: The TDF can be used to examine the barriers and facilitators to nutritional risk screening of older adults in primary care. Identification and classification of these barriers and facilitators can aid in the development and implementation of interventions designed to improve rates of nutritional risk screening in primary care. Identifying older adults at nutritional risk can help to prevent malnutrition, by intervening early when poor dietary intake may still be relatively easy and inexpensive to address. Screening is the first step in this identification.

\section{Conflict of Interest}

There is no conflict of interest 